\title{
Immune checkpoint inhibitors in lymphoma: challenges and opportunities
}

\author{
Haris Hatic ${ }^{\#}$, Devi Sampat”, Gaurav Goyal^^ \\ Division of Hematology-Oncology, University of Alabama, Birmingham, AL, USA \\ Contributions: (I) Conception and design: G Goyal; (II) Administrative support: G Goyal; (III) Provision of study material or patients: None; (IV) \\ Collection and assembly of data: H Hatic, D Sampat; (V) Data analysis and interpretation: All authors; (VI) Manuscript writing: All authors; (VII) \\ Final approval of manuscript: All authors. \\ "These authors contributed equally to this work. \\ Correspondence to: Gaurav Goyal, MD. Division of Hematology-Oncology, The University of Alabama at Birmingham, 1802 6th Avenue South Suite \\ 2555, Birmingham, AL 35294, USA. Email: ggoyal@uabmc.edu.
}

\begin{abstract}
Immune checkpoint inhibitors (ICIs) are immunomodulatory antibodies that intensify the host immune response, thereby leading to cytotoxicity. The primary targets for checkpoint inhibition have included cytotoxic T-lymphocyte-associated antigen 4 (CTLA-4), programmed cell death receptor-1 (PD-1) or programmed cell death ligand-1 (PD-L1). ICIs have resulted in a change in treatment landscape of various neoplasms. Among hematologic malignancies, ICIs have been most successful in certain subtypes of lymphomas such as classic Hodgkin lymphoma (cHL) and primary mediastinal B-cell lymphoma (PMBCL). However, there have been several challenges in harnessing the host immune system through ICI use in other lymphomas. The underlying reasons for the low efficacy of ICI monotherapy in most lymphomas may include defects in antigen presentation, non-inflamed tumor microenvironment (TME), immunosuppressive metabolites, genetic factors, and an overall lack of predictive biomarkers of response. In this review, we outline the existing and ongoing studies utilizing ICI therapy in various lymphomas. We also describe the challenges leading to the lack of efficacy with ICI use and discuss potential strategies to overcome those challenges including: chimeric antigen receptor T-cell therapy (CAR-T therapy), bispecific T-cell therapy (BiTE), lymphocyte activation gene-3 (LAG-3) inhibitors, T-cell immunoglobulin and mucindomain containing-3 (TIM-3) inhibitors, vaccines, promotion of inflammatory macrophages, indoleamine 2,3-dioxygenase 1 (IDO1) inhibitors, DNA methyltransferase inhibitors (DNMTi) and histone deacetylase inhibitors (HDACi). Tumor mutational burden and interferon-gamma release assays are potential biomarkers of ICI treatment response beyond PD-L1 expression. Further collaborations between clinicians and scientists are vital to understand the immunopathology in ICI therapy in order to improve clinical outcomes.
\end{abstract}

Keywords: Hodgkin; pembrolizumab; nivolumab; ipilimumab; cytotoxic T-lymphocyte-associated antigen 4 (CTLA-4)

Submitted Oct 11, 2020. Accepted for publication Jan 25, 2021.

doi: 10.21037/atm-20-6833

View this article at: http://dx.doi.org/10.21037/atm-20-6833

\footnotetext{
^ ORCID: 0000-0001-6148-5177.
} 


\section{Introduction}

The use of immune checkpoint inhibitors (ICIs) has ushered in a paradigm shift in oncology due to successful treatment in various malignancies. After tremendous successes in metastatic melanoma, ICIs were explored in hematologic and other solid organ malignancies. Among solid tumors, ICIs have been approved for treatment of melanoma, renal cell carcinoma, lung cancer, head and neck cancer, gastric cancers, triple-negative breast cancer, urothelial carcinoma, hepatocellular carcinoma, cervical cancer, ovarian cancer, and colorectal cancer (1). However, the efficacy of ICIs in hematologic malignancies has been limited, and mostly seen in certain subtypes of lymphoma (2).

ICIs are immunomodulatory antibodies that intensify the immune system, activate $\mathrm{T}$ cell function, and aid in cancer cell death (3). Normally, T-cell activation occurs due to an inciting event such as an infection, inflammation, or malignancy. T-cell activation occurs through the presentation of antigens that are bound on T-cell receptor (TCR) and major histocompatibility complex (MHC) on the surface of antigen-presenting cells (APCs) (4). Next, a complex cascade of pathways is activated, whereby an antigen attaches to a TCR with an ensuing co-stimulation of immune checkpoints in order to provide suppression of the immune response or manage cytotoxicity (5). The balance between the stimulatory and inhibitory signals is mediated via specific membrane receptors or ligands on T-cell surface (6). These specific ligands include cytotoxic T-lymphocyte-associated protein 4 (CTLA4) and programmed cell death-1 (PD-1) (7).

\section{Mechanism of action}

To discern the mechanism of action of ICIs, it is crucial to understand how the CTLA-4 and PD-1 ligands play a role in the immune response. CTLA-4 regulates T-cell proliferation early in an immune response, primarily in lymph nodes, whereas PD-1 suppresses T-cells later in an immune response, primarily in peripheral tissues (4). CTLA-4 works by indirectly diminishing signaling through its co-stimulatory receptor, CD28 (Figure 1). As such, CTLA-4 increases the activation threshold of $\mathrm{T}$ cells, reducing immune responses to weaken antigens such as self- and tumor antigens (7). PD-1 attaches to programmed death-ligand 1 (PD-L1), a protein on some normal (and cancer) cells. The PD-1/PD-L1 interaction then inhibits T-lymphocyte proliferation, survival and effector functions such as cellular toxicity and cytokine release (7).

Various ICIs are available that target specific immune checkpoints: anti-CTLA-4 (ipilimumab), antiPD-1 (nivolumab, pembrolizumab), and anti-PDL-1 (atezolizumab, avelumab, and durvalumab). Ipilimumab was the first ICI to be approved in oncology due to robust responses demonstrated in metastatic melanoma (8). While most responses with ICIs were reported in solid organ malignancies, lymphomas have the most outcomes data among the hematologic neoplasms. In May 2016, nivolumab was approved for treatment of classic Hodgkin lymphoma (cHL) in patients with relapse after autologous hematopoietic stem cell transplant (AHSCT) (9). The following year in March 2017, pembrolizumab was approved for the treatment of relapsed/refractory classic $\mathrm{HL}(\mathrm{r} / \mathrm{r} \mathrm{cHL})$ after 3 or more prior lines of therapy, and most recently in 2020 this was extended to failure of one line of therapy (9). The only other approved indication of ICIs in lymphoma is pembrolizumab in primary mediastinal B-cell lymphoma (PMBCL) after failure of 2 or more lines of therapy. Over the last few years, our understanding of the immune biology and role of ICIs in lymphoma has grown.

In this review, we provide a comprehensive overview of the extant literature using ICI therapy in lymphoma, with focus on key endpoints like overall response rate (ORR), progression-free survival (PFS), and overall survival (OS). We also discuss the challenges associated with ICI therapy in lymphomas, and the strategies to overcome those challenges in order to improve their efficacy.

\section{Efficacy of ICls in lymphoma}

\section{cHL}

Around $80 \%$ of patients with cHL can be cured by first line therapy alone; however, challenges in $\mathrm{cHL}$ arise when selecting what agents should be used for refractory or relapsed disease. Among lymphomas, ICIs have demonstrated robust responses particularly in cHL (10). The reasons for the remarkable responses seen with ICI therapy in cHL are potentially multifold. The Reed Sternberg cells (RSC) of cHL attract a rich immune infiltrate of CD4/CD8 cells surrounding them. RSC are characterized by two major immune pathways: nuclear factor kappa-light-chain-enhancer of activated B cells (NF$\mathrm{kB}$ ) and Janus kinase (JAK) pathways which further amplify PD-L1 expression (10). It is also important to note that the chromosomal region 9p24.1 that contains both the 


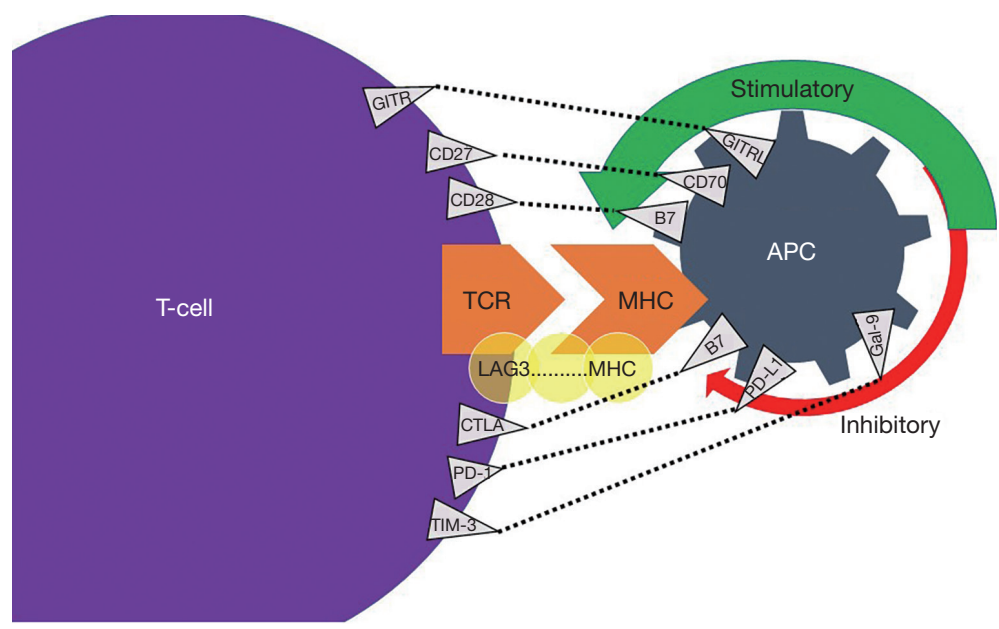

Figure 1 Inhibitory \& stimulatory T-cell cognate ligand receptors. Some known stimulatory and inhibitory ligands on TCR. Some of the upregulators of T-cells and their cognate ligand are CD27-CD70, GITR-GITRL, CD28-B7, ICOS-ICOSL. Inhibitory TCRs and their ligand include: LAG3-MHC, CTLA4-B7, PD1-PDL1, TIM3-Gal9. CTLA 4, cytoxic T-lymphocyte associated protein 4; GITR, glucocorticoid-induced tumor necrosis factor receptor; GITRL, glucocorticoid-induced tumor necrosis factor receptor ligand; MHC, major histocompatibility complex; LAG3, lymphocyte-activation gene 3; PD-1, programmed cell death protein 1; PDL-1, programmed death ligand 1; TIM-3, T cell immunoglobulin and mucin domain-containing protein 3; TCR, T-cell receptor; Gal9, galectin-9.

PD-L1 and PD-L2 genes is amplified in RSCs, resulting in overexpression of both proteins. Another theory for high efficacy of ICIs in cHL is based on its association with Epstein-Barr virus (EBV). EBV infection in cHL might potentially increase the PD-L1 expression such that blocking the PD-1 and PDL-1 pathway may restore immunosurveillance in cHL (10).

At least four different phase I-II trials have showed ORR of $>70 \%$ using anti PD-1 therapy (10-13). The first landmark study by Ansell et al. enrolled 23 patients with heavily pre-treated relapsed or refractory $\mathrm{cHL}$ to receive nivolumab, and demonstrated an ORR of $87 \%$, including $17 \%$ with a complete response (CR) and $70 \%$ with a partial response (PR) (10). The rate of progression-free survival at 24 weeks was $86 \%$ (10). Subsequent studies using nivolumab, pembrolizumab or a combinational therapy with PD-1 and CTLA-4 blocking agents showed sustained remissions in responders (Table 1) (14-16). A recent study showed superior PFS with the use of pembrolizumab as compared with brentuximab vedotin for $\mathrm{r} / \mathrm{r} \mathrm{cHL}$ after one line of therapy among patients ineligible for AHSCT (38). These studies have resulted in regulatory approval of nivolumab after 2 lines of treatment including autologous stem cell transplant and brentuximab vedotin, and of pembrolizumab after one line of therapy. In a phase I/II trial evaluating the efficacy of nivolumab and brentuximab vendotin on refractory/relapsed cHL, $61 \%$ of patients achieved a CR, with an ORR of $82 \%(\mathrm{~N}=62)(12)$. Due to the high response rates, ongoing studies are evaluating the role of ICIs in combination with chemotherapy in the frontline or salvage therapy of cHL (Table 2).

\section{Diffuse large B-cell lymphoma (DLBCL)}

Anti-PD-1 antibodies pembrolizumab and nivolumab have been evaluated in DLBCL as well, though with suboptimal responses. PD-L1 overexpression is observed in about of $20 \%$ of DLBCL and occurs primarily on macrophages (39). Kiyasu et al. examined over 1,250 DLBCL samples using PD-L1 and PAX5 staining techniques and found that 10.5\% of patient specimens expressed PD-L1 and this increased to about $15 \%$ when the microenvironment was included with a threshold for PD-L1 positivity set at 30\% (40). The variability of PD-L1 expression in DLBCL is dependent on the subtype and threshold of PD-L1 positivity. Structural changes of 9p24.1 leading to PD-L1 expression are seen in $10 \%$ of patients, and mostly in the non-germinal center type of DLBCL (41). However, there have been several studies involving ICI treatments in DLBCL with varied results (Table 1) (13,17-21). Although the initial phase I study showed an ORR of $36 \%$ with nivolumab (21), subsequent phase II studies revealed a dismal ORR $<10 \%$ 


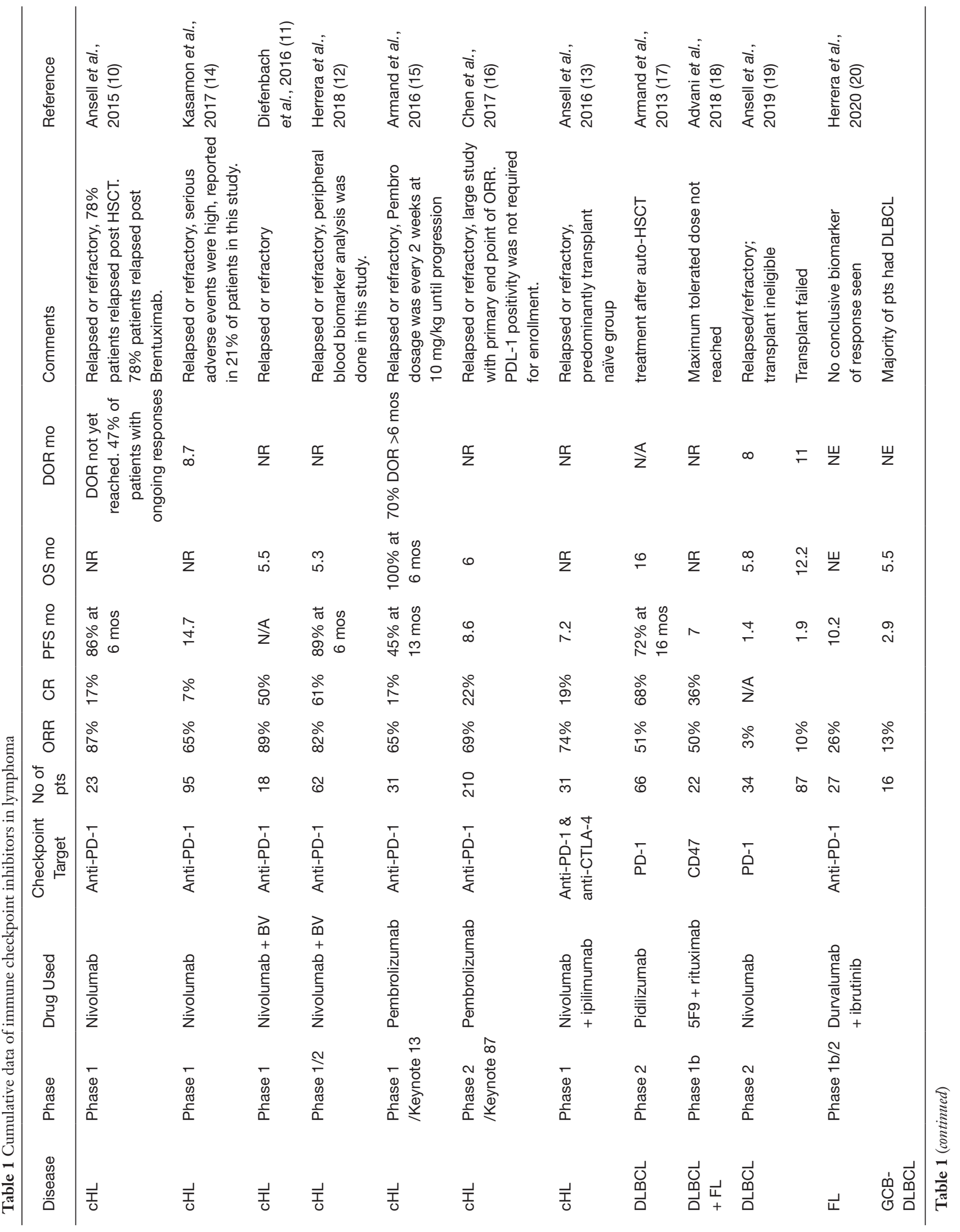




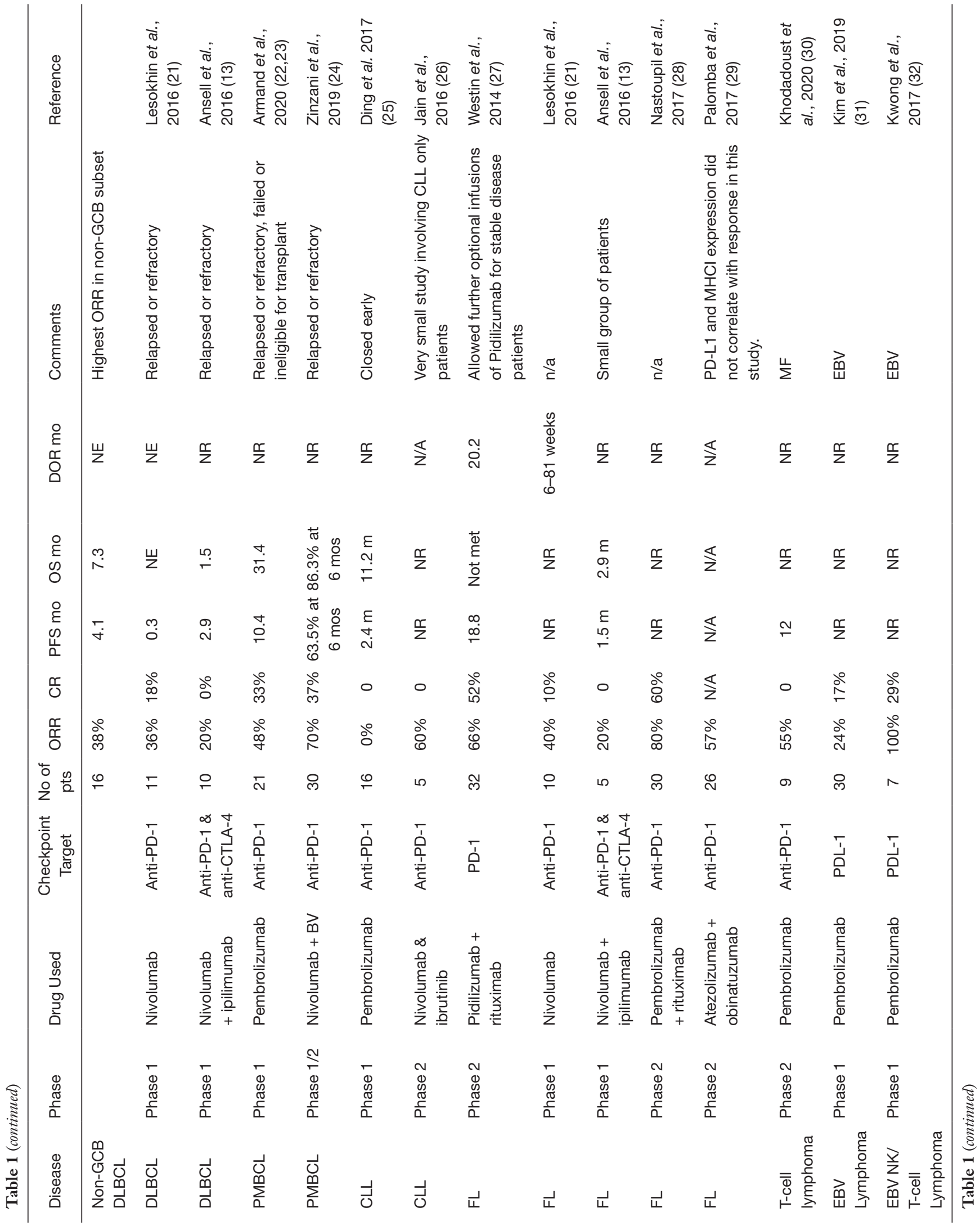




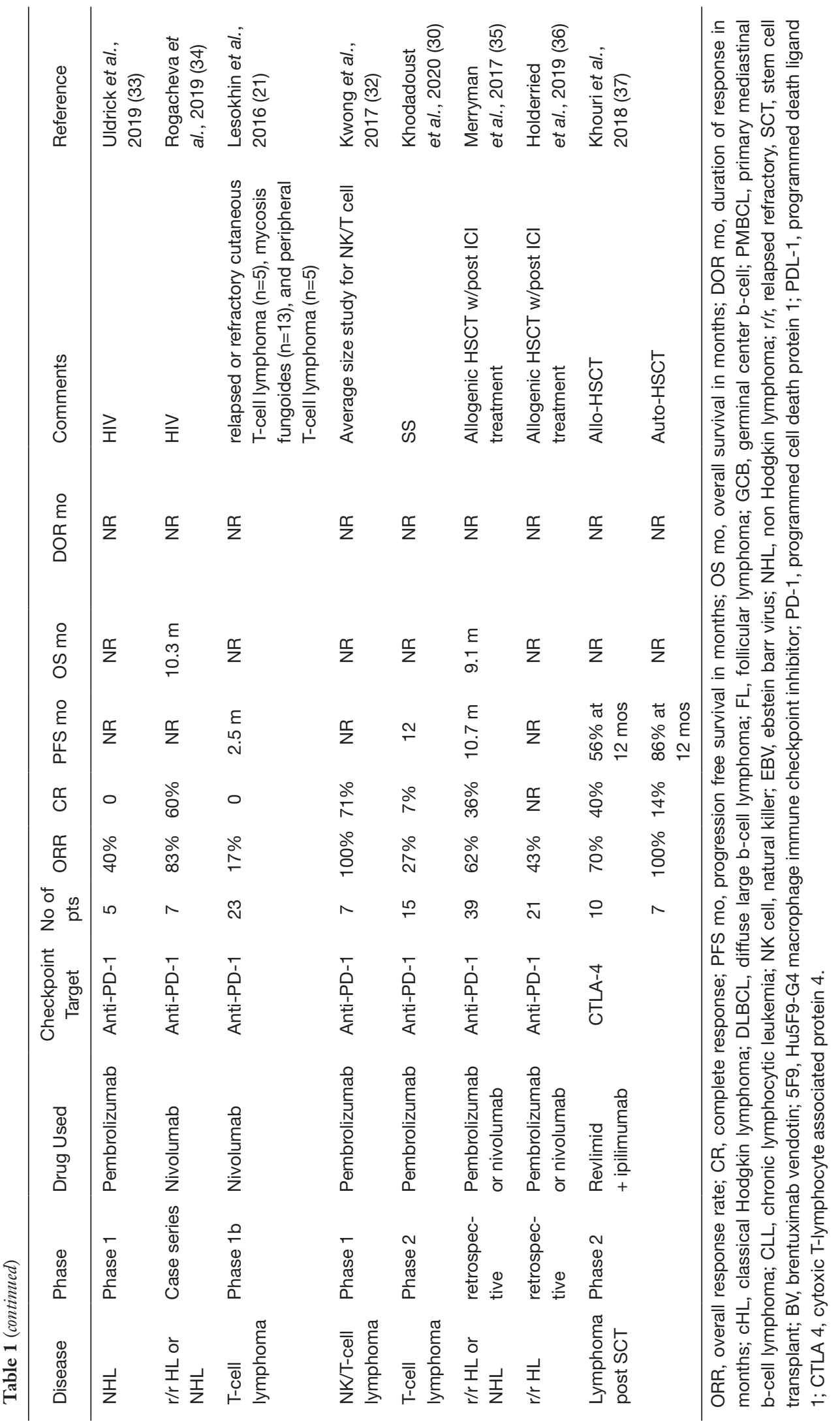




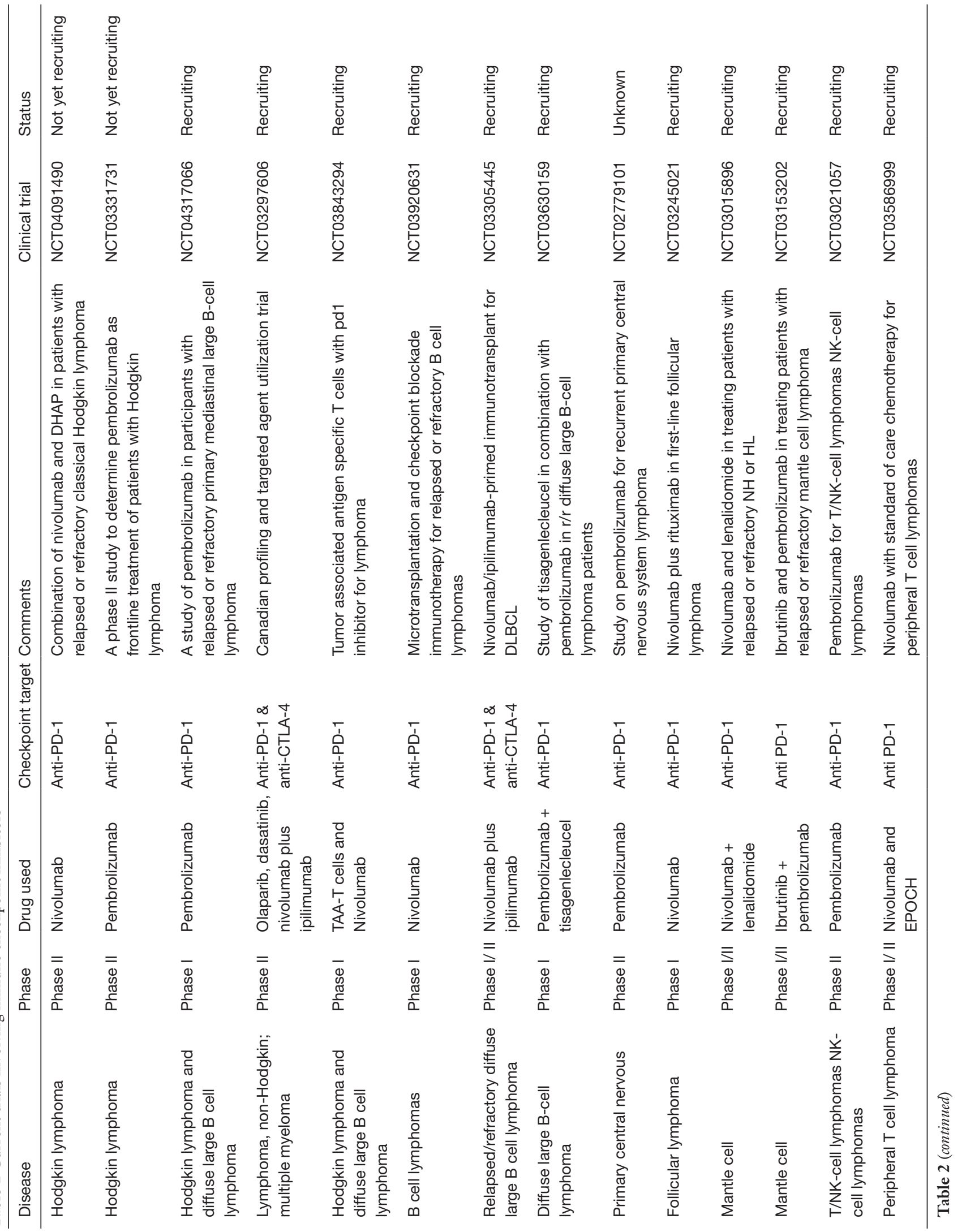


with ICI monotherapy (Table 1). Although there are several ongoing studies examining the combination of ICIs with other agents in DLBCL, there is a need to identify predictive biomarkers to identify patients that will benefit from such an approach.

\section{PMBCL}

ICI therapy has also been evaluated in PMBCL, which is the only other lymphoma subtype than cHL to show robust responses with ICI therapy. This is perhaps in part because PMBCL frequently expresses PD-L1/2, which is not seen in most other mature B-cell lymphomas. Approximately 30-80\% of patients with PMBCL have PD-L1 overexpression (42). PMBCLs also share pathologic and genetic features with cHL. In a phase I study (Keynote 013), the ORR with anti PD-1 therapy in relapsed/refractory PMBCL was $48 \%$, with median OS of 31.4 months (22) (Table 1). This study led to the approval of pembrolizumab by the US-FDA in PMBCL patients after failure of 2 or more lines of therapy. Another trial utilized a combination of nivolumab and brentuximab vedotin, with ORR of $70 \%$, CR of $37 \%$, and median OS not reached at 11 months follow-up (24). However, both these trials had a small sample size, and there is a need for larger studies as well as long-term follow-up data supporting ICI use in this disease.

\section{Primary central nervous system lymphoma (PCNSL) and testicular lymphoma (PTL)}

PCNSL and PTL demonstrate high expression of PD-L1 and PD-L2 through amplification 9p24.1, which makes it attractive to use ICI therapy (43). Nivolumab was tested in four patients with PCNSL and one patient with PTL in the relapsed/refractory setting. Interestingly, all five patients had an objective response, with three patients in ongoing remission over one year. There is a current phase II study underway with pembrolizumab in relapsed/refractory PCNSL (Table 2, NCT02779101).

\section{Chronic lymphocytic leukemia/small lymphocytic lymphoma (CLL/SLL) and richter transformation}

In CLL/SLL, anti-PD1 therapy using pembrolizumab has been examined in various trials. PD-L1/PD-1 expression in CLL/SLL can range between 10-90\% $(44,45)$. Effector memory T-cells in CLL are impaired, thus cannot form immune synapse with CLL cells which is reflected in 
PD-L1 overexpression in the tumor microenvironment (TME) of histiocytes (46). This large variability of PD-L1 expression is likely secondary to differences in antibodies used, methodology employed, and staining specificity (47). Nevertheless, in aggressive cases of CLL/ SLL, there is an expansion of CD8+/PD-1 and T memory cells that subsequently inverts the CD4:CD8 ratio (48). It seems that increased PD-L1 expression on T-cells had no prognostic significance (49). In a phase II study, pembrolizumab was administered to relapsed CLL and Richter transformation (RT) patients (25). The study demonstrated an ORR of $44 \%$ and a median OS of about 11 months among RT cohort (Table 1). However, no responses were seen among CLL cohort in this study. In this study, higher PD-L1 expression on tumor cells and microenvironment was associated with a response to ICIs in RT (25). There is some evidence to suggest that using BTK inhibitors before ICIs may increase the efficacy of ICIs, though further studies are needed to confirm this finding $(26,50)$.

\section{Follicular lymphoma (FL)}

The expression of PD-1 and PDL-1 on tumor cells of FL is rare. Several studies have examined the role of ICIs in relapsed/refractory FL (Table 1) (13,21,27-29). In an openlabel non-randomized trial of 32 patients with relapsed FL, pidilizumab was administered with rituximab weekly for 4 weeks (27). The ORR was $66 \%$ and CR was $52 \%$ with median PFS of 18.8 months (27). In another phase Ib trial, 5 patients with relapsed/refractory non-Hodgkin lymphoma (NHL) received nivolumab plus ipilimumab followed by nivolumab monotherapy (13). In this study, the ORR was $20 \%$, with a PFS of 1.5 months and OS of 2.9 months. However, when studied in phase II trial of 92 patients, the ORR was $4 \%$, with median PFS of 2.2 months with nivolumab monotherapy (51).

\section{Mantle cell lymphoma (MCL)}

While preclinical studies have suggested a mechanistic role for ICIs in patients with MCL, clinical data thus far suggests only modest success. The expression of therapeutically targetable immune checkpoint molecules has been analyzed on primary MCL cells. MCL cells showed constitutive expression of PD-1 and PDL-1, but absence of PD-L2 and CTLA-4 (52). Furthermore, it was found that induction of PD-L1 was attenuated by concurrent treatment with ibrutinib or duvelisib, suggesting BTK and PI3K are important mediators of PD-L1 expression (52). To date, there are no completed trials of immunotherapy treatments specifically for MCL patients, however, several trials have included a small subset of MCL patients to assess efficacy of ICIs. In a phase I trial of nivolumab in relapsed/refractory NHLs, no responses were seen among the four patients with MCL (21). An ongoing trial is using lenalidomide and nivolumab to assess safety and response in patients MCL (Table 2). Another clinical trial is looking at BTK inhibitor combined with pembrolizumab for relapsed or refractory MCL (Table 2).

\section{Burkitt lymphoma (BL)}

$\mathrm{BL}$ is well known to be a highly aggressive lymphoma in which rapid, high intensity chemotherapy is standard of care. Typically, aggressive chemotherapy regimens used for this disease can put young, functional patients in complete remission with low proportion of primary refractory disease. ICIs have not been utilized outside the context of clinical trials for these patients. The ongoing trial that has been partly reported involves varlilumab or CDX1127, a fully human monoclonal antibody that binds to a molecule called CD27 found on T-cells and also on certain hematologic tumor cells to promote anti-tumor effects. The trial initially assessed safety in phase I, and it has now moved onto phase II utilizing nivolumab with or without varlilumab in aggressive b-cell lymphomas, including $\mathrm{BL}$ (Table 2).

\section{Epstein Barr virus (EBV)-associated lymphoma}

EBV, or human herpesvirus, has infected over $90 \%$ of adults worldwide and remains lifelong in the latent phase (53). In a small percentage of patients, it can lead to BL, cHL, lymphoproliferative disease (LPD) in immunodeficient individuals, and DLBCL (54). Latent EBV infections can transmit through infected tumor cells and cause inflammation in the TME. In cHL associated with EBV, dendritic cells (DCs) and tumor associated macrophages (TAM) are detected in tumor specimens (55). In extranodal NK/T-cell lymphoma, nasal type (ENKL), the EBV activates latent membrane protein-1 (LMP1) which induces NF-kB to produce T-helper cell-1 (TH1) cytokines tumor necrosis factor-alpha (TNF- $\alpha$ ) and interferon gamma (IFN- $\gamma$ ) (56). In ENKL there is an upregulation of PD1 in order to suppress $\mathrm{T}$ cell toxicity (56). EBV infection has 
been reported to upregulate PDL-1 expression and PD-L1/ PD-L2 gene alterations in a large proportion of lymphomas (57-59). For instance, in DLBCL, PD-L1 was expressed in about $60 \%$ of cases and on average $20 \%$ of patients had a PD-L1/PD-L2 genetic expression in EBV-positive lymphoma $(58,60)$.

In a study of relapsed or refractory NK/T cell lymphoma using pembrolizumab (Table 1), CR rate was $71.4 \%$ and two patients had molecular remission (32). In another trial, seven patients with EBV+ NHL showed a response including NK/T cell lymphoma (44\%) and primary mediastinal B cell lymphoma (25\%). EBV-negative subtypes of DLBCL and T-lymphoblastic lymphoma did not respond (31). Also, PD-L1 expression was $56 \%$ in $\mathrm{EBV}+$ compared to EBV- patients whose expression level was $11 \%(31)$.

\section{Human immunodeficiency virus (HIV)-associated- lymphoma}

Antiretroviral therapy (ART) has dramatically improved HIV management and outcomes. Interestingly, CTLA4 and PD-1 expression tends to correlate with HIV viral load, number of CD4+ cells, and disease progression (61). In a small study, it was found that HIV RNA increased with ICI therapy due to latency reversal (4). PD-L1 expression is the highest in HIV patients with or without an EBV co-infection in B-cell lymphoma (62). Most trials of ICIs exclude people living with HIV, which make the use of ICIs a challenge as questions related to side-effects, medication interactions and outcomes remain unanswered.

A recent phase I trial (Table 1) examined the safety profile of pembrolizumab for HIV malignancies (33). Of the five patients with NHL in the trial, PRs were seen in four patients (including one patient with primary effusion lymphoma) (33). No unique toxicities were reported in this cohort. These results showed that ICIs can be used safely among people living with HIV and cancer. In a case series, nivolumab use was reported as salvage therapy in relapsed/refractory cHL $(n=4)$ and NHL $(n=2)(34)$. In the case series, two patients received monotherapy (both cHL) with nivolumab, while the rest received the combination of nivolumab with bendamustine and gemcitabine. The ORR was $83 \%$, and $60 \%$ achieved a CR (Table 1). Among the two patients with cHL who received nivolumab monotherapy, one achieved a CR and other a PR. These limited data are a first crucial step in the investigation of ICIs among people living with HIV and lymphoma.

\section{T-cell lymphoma}

Although ICI therapy relies on activation of exhausted T-cells, there are data to suggest that tumor cells of T-cell lymphomas express PD-1, with frequent copy number losses of PD-1 in cutaneous T-cell lymphomas (63). In an early phase basket study, the ORR rates using nivolumab were $15 \%$ and $40 \%$ among patients with mycosis fungoides and peripheral T-cell lymphomas (PTCL), respectively (21).

A subsequent study evaluating outcomes among PTCL using nivolumab was presented at American Society of Hematology meeting in 2019. Unfortunately, hyperprogression (defined as dramatic progression within 1 cycle of treatment) was noted in one-third patients $(n=4)$, which led to halting of the study (64). In a phase II trial of pembrolizumab among patients with advanced Sezary syndrome, ORR was $38 \%$ with two CRs and seven PRs. Of the responding patients, six had $90 \%$ or more improvement in skin disease (30). Therefore, ICI therapy deserves further exploration in cutaneous T-cell lymphomas (Table 1) $(21,30,32)$.

\section{Autologous/allogenic hematopoietic stem cell transplantation}

Given the immune remodeling and low tumor burden after AHSCT, various studies have examined the role of ICIs post-AHSCT in lymphoma. In a phase II study, pidilizumab (anti PD-1 antibody) was utilized postAHSCT in DLBCL patients (17). Among the 35 patients with measurable disease post-AHSCT, the ORR was $51 \%$. Among the entire cohort, the PFS at 16 months was $72 \%$, suggesting a potential role of ICI therapy in this setting especially in high-risk patients (17). Another vital question with use of ICIs arises in the allogeneic hematopoietic stem cell transplant (HSCT) setting, due to concerns for potential worsening of graft-versus-host-disease (GVHD). In a multicenter retrospective analysis of 39 lymphoma patients $(79 \% \mathrm{cHL})$ who received ICI treatment prior to allogeneic HSCT, the ORR with PD-1 blockade was $78 \%$, with $41 \%$ CRs (35). The two-year OS and PFS were $79 \%$ and $65 \%$, respectively (35). The one-year cumulative incidence of grade 3-4 acute GVHD was $23 \%$, and that of chronic GVHD was $41 \%$ (35). There are several studies that indicate an increase in GVHD due to enhancement from the IFN- $\gamma$ dependent mechanism (65-67). In a multicenter retrospective study of 31 lymphoma patients (94\% cHL) who received ICI therapy post-allogeneic HSCT, 
$55 \%$ patients developed treatment-emergent GVHD that was refractory to most treatments in majority of cases (66). Further studies are required to confirm these findings with a longer follow-up periods that examine outcomes, complications and risk factors for toxicity (Table 1) (35-37).

\section{Challenges limiting the efficacy of ICI therapy in Iymphoma}

\section{Antigen presentation}

Defective antigen presentation is likely the leading factor and explanation for reduced efficacy of ICI therapy specifically in lymphomas. This is because it is common to have a loss of MHC-I on the lymphoma cell surfaces, ultimately due to irreversible mechanisms such as alterations in the MHC-I gene itself and mutations in the B2-microglobulin $(\beta 2 \mathrm{M})$. On a broad level, lymphomas can evade the immune system recognition through the downregulation of molecules involved in antigen presentation (68). Loss of MHC-I on the surface of CD8+ cells occurs in about $60 \%$ of DLBCL and cHL (69). The main reason for this is a loss-of-function mutation of the $\beta 2 M$ which occurs in about $20 \%$ of $\mathrm{FL}, 30 \%$ of $\mathrm{BL}, 30 \%$ of DLBCL, $50 \%$ of PMBCL, and at least $50 \%$ of $\mathrm{HL}$ (70-72). Likewise, downregulation of MHC-II occurs via mutations in the class II transactivator (CIITA) (73). Defective antigen presentation can also be caused by gamma-interferon-inducible-lysosomal thiol reductase (GILT) and human leukocyte antigen DM (HLA-DM) (74). These two enzymes of the antigen processing machinery are downregulated by c-MYC (74). Apart from further research to overcome this immune evasion, a potential alternative would be monotherapy or combination therapy with MHCindependent treatments such as chimeric antigen receptor T-cell therapy (CAR T-cell therapy) or bispecific T-cell engager antibodies (BiTEs) (Figure 2). CAR-T therapy uses a patient's own T-cells to attack the tumor cells via a CAR molecule, and have been approved for relapsed/ refractory DLBCL and MCL. BiTEs represent a bridge by targeting an antigen on lymphoma and another on T-cells in order to induce cell-mediated cytotoxicity. In a phase I trial of relapsed/refractory B-cell NHL patients using BiTE antibody, there was an ORR of $69 \%$ and CR $37 \%$ with median duration of response (DOR) of 17 months (75).

Combinational treatment using ICIs can be approached in two specific ways. First, ICIs can be combined with chemotherapy, monoclonal antibody, targeted therapy, or
CAR-T cell therapy in order to induce antigen presentation by APC (76). Some of the combination trials with ICIs include ibrutinib (NCT02950220, NCT02940301), PI3K inhibitors (NCT03471351), lenalidomide (NCT02875067), and anti-CD20 antibody (NCT03121677) (20,21). The second way that combinational treatment can be utilized is through the use of two ICIs which can potentially overcome the issue of defective antigen presentation by enhanced T-cell activation. However, special care should be taken to avoid lymphocyte depleting regimens as they can be counter-productive in this setting.

\section{TME and tumor associated macrophages}

The TME may also determine the immune response to ICI therapy. Most lymphomas, such as DLBCL, FL, CLL, and BL harbor a "noninflamed" microenvironment which is defined by a low infiltration of immune cells with a plethora of genetic escape alterations (68). These excluded immune cells can further promote tumor proliferation through cytokines, chemokines, myeloid-derived suppressor cells (MDSCs), and tumor-infiltrating lymphocytes (TILs) (77).

T-cell response is also dependent on co-receptors (Figure 1). Some of the upregulators of T-cells and their cognate ligand are CD27-CD70, GITR-GITRL, CD28-B7, ICOS-ICOSL, CD137-CD137L, and OX40-OX40L (78). Inhibitory TCRs and their receptive cognate ligand include: LAG3-MHC, CTLA4-B7, PD1-PDL1, TIM3Gal9, and BTLA-HVEM (78). PD-1 blockade resistance can occur via lymphocyte activation gene-3 (LAG-3) and $\mathrm{T}$ cell immunoglobulin and mucin-domain containing-3 (TIM-3) (79). LAG-3 is a competitor of CD4 and binds to MHC-II. In EBV+ cHL, LAG-3 is expressed on the $\mathrm{T}$ cells and associated with reduced CD8+ response which is currently being examined in a series of phase I/ II trials (79-81) (NCT03311412). Also, in lymphoma TIM-3 upregulation on CD8+ T-cells and TILs leads to tumor resistance, which is a process that could be reversed with combinational blockade of PD-1 and anti-TIM-3 monoclonal antibodies (82) (NCT03489343).

In relapsed cHL, there are increased number of PD-1 positive $T$ cells and continuous TCR stimulation from high antigen exposure can lead to exhaustion of effector T-cells (83). Modulating the TME can be a solution to how this resistance could be overcome. A CTLA-4 inhibitor could be used to induce regulatory $\mathrm{T}$ cell (Treg) depletion (84). Treg infiltration can be reduced by a low dose of cyclophosphamide through downregulation of 


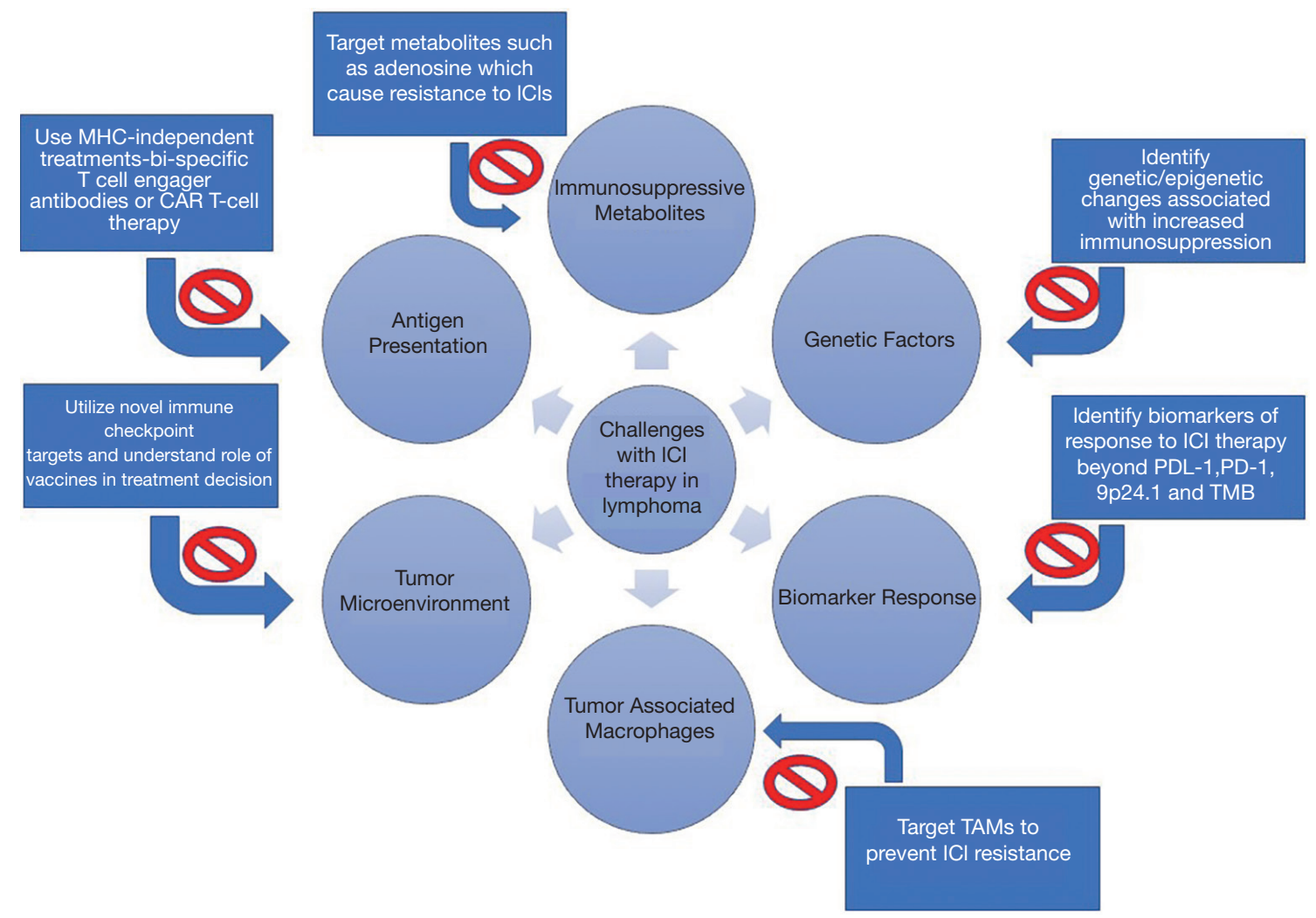

Figure 2 Challenges with immune checkpoint inhibitors therapy and strategies to overcome those challenges. There are six specific challenges with immune checkpoint inhibitors (ICIs) and they include: antigen presentation, tumor microenvironment (TME), tumor associated macrophages (TAM), immunosuppressive metabolites, genetic factors and biomarker response. For each of the challenges specific strategies are described that can overcome it. For antigen presentation the use of major histocompatibility complex independent treatment can be used such as chimeric antigen receptor T-cell therapy (CAR T-cell therapy) or Bispecific T-cell engager antibodies (BiTE). For TME challenges, novel checkpoint inhibitors can be used such as lymphocyte activation gene-3 (LAG-3) and T cell immunoglobulin and mucin-domain containing-3 (TIM-3) inhibitors with programmed death ligands. Vaccines are also investigated to overcome this challenge. Use of anti-CSF antibodies or the promotion of inflammatory macrophages through phosphatidyl 3-kinase- $\gamma$ inhibitors can counteract TAMs resistance. Immunosuppressive metabolite such as indoleamine 2,3-dioxygenase 1 (IDO1) can be bypassed by an inhibitor such as epacadostat. Microarrays can identify specific genes much easier and allow of analysis of the TME for assessment of immune evasion. Epigenetic therapies can overcome some of those changes through DNA methyltransferase inhibitors (DNMTi) and histone deacetylase inhibitors (HDACi). Lastly, newer biomarkers are being identified such as serum IFN- $\gamma$ levels and number of CD8-positive monocyte tumor infiltrating lymphocytes (TILs) on the tumor sample. ICI, immune checkpoint inhibitor; CAR-T, chimeric antigen receptor T cell; MHC, major histocompatibility complex; SNP, single nucleotide polymorphisms; PD-1, programmed cell death protein 1; PDL-1, programmed death ligand 1; TMB, tumor mutational burden; TAM, tumor associated macrophages; IO, immunotherapy.

forkhead box P3 (FOXP3) (85).

TAMs promote immunosuppression and phagocytosis. They are divided into M1 (pro-inflammatory) and M2 (antiinflammatory) subsets. M2 macrophages are CD163+ and are recruited by upregulation of interleukin 10 (IL-10) (86). In cHL, the presence of CD163+/CD68+ macrophages in the TME was found to be associated with shorter survival and chemotherapeutic resistance (87). TAMs seem to influence CD4+ T-cell dysfunction by preventing access of HRS cells through the PD-1-PD-L1 interaction (87). The combinational use of PD-1 blockade with macrophage depleting therapies such as anti-CSF antibodies or the promotion of inflammatory macrophages through phosphatidyl 3-kinase- $\gamma$ inhibitors can counteract TAMs 
resistance (68).

In lymphoma, we know that tumor vaccines are unable to induce responses, likely due to antigen-TME mismatch (88). One specific target that has emerged in vaccine therapy is the immunoglobulin idiotype (Id) (89). Id peptides can be combined to DNA or protein which causes a DC response in vivo (90). Another approach is to load the DNA or protein into DCs. Subsequently, this causes an immune response as DCs generate a specific tumor antigen (90). In one study, 287 patients with untreated FL were randomized 2:1 to 7 months of a protein-based vaccine or placebo after achieving a response (91). The primary endpoint of the study was PFS, and was not significantly different in the two arms at a median follow-up of 58 months, although the PFS among patients who had a humoral response was significantly higher than those who did not. For DC loaded vaccines an Id based protein with tumor lysate was used to illicit an immune response. A group of 18 patients with relapsed indolent B-cell NHL were given a DC-based vaccine with ORR of $33 \%$ and 3 CRs (92). An ongoing phase I trial with FL patients examines the use of a personal vaccine with the goal of broad activation of innate and adaptive immunity with nivolumab (NCT03121677). Future approaches related to vaccines will require a better understanding of the TME in order to enhance immunogenicity via novel nanoparticle delivery systems to the tumor-draining lymph nodes (93).

\section{Immunosuppressive metabolites}

There are several immunosuppressive metabolites that can contribute to ICI resistance. Adenosine is a molecule that suppresses effector $\mathrm{T}$-cell and increases $\mathrm{T}$ regulator (Treg) cells through the A2a receptor (86). Once bound, the receptor enables the tumor proliferation by reducing the activity of DCs, natural killer cells (NKCs), M1 macrophages, and CD8+ T-cells (86). In cHL, elevated production and reduced degradation lead to increased adenosine levels and reduced PD-1 blockade (94). Another immunosuppressive metabolite is indoleamine 2,3-dioxygenase 1 (IDO1). IDO1 is an enzyme that converts tryptophan to a metabolite called kynurenine in the TME which causes T-cell anergy and apoptosis (95). This metabolite may be a cause of resistance to ICIs via suppression of $\mathrm{T}$ effector cell function. IDO1 inhibitor epacadostat (NCT03322384) is currently being investigated in lymphoma as a synergistic cancer therapeutic agent with ICIs that could overcome immunosuppressive metabolites (96).

\section{Genetic factors}

The immune escape that renders ICIs ineffective in certain lymphomas is driven by different genetic and epigenetic factors in the tumor cells and TME. Alteration in the oncogenic and tumor suppressor genes such as phosphate and tensin homolog $(P T E N)$, enhancer of zeste homolog 2 (EZH2), MYC and TP53 causes immune cell exclusion and suppression (68). As a result, there is decreased expression of the innate immune system. For instance, PTEN loss without phosphatidyl inositol 3-kinase catalytic subunit (PIK3CA) mutations activates the product of class I phosphoinositide 3-kinase (PI3K) in MCL (97). EZH2 inhibits HLA expression in DLBCL and $M Y C$ which cause changes in the transcriptional signatures and mutations in NF- $\kappa B$ pathway $(98,99)$. Recent microarray technologies have made the identification of specific genes much easier and allow of analysis of the TME for assessment of immune evasion (100). Epigenetic changes such as DNA methylation and histone post-translational modifications can also cause ICIs resistance (101). In CLL, hypomethylations in the promoter region caused PDL-1 elevations in the protein levels (102). Epigenetic therapies can overcome some of those changes through DNA methyltransferase inhibitors and histone deacetylase inhibitors (103).

\section{Biomarkers of response}

There is a need to develop biomarkers that can predict response to ICI therapy in lymphomas. In cHL and $\mathrm{PMBL}$, there is an increased $\mathrm{PD}-\mathrm{L} 1 / \mathrm{L} 2$ protein expression and it can be indicative of a durable PFS (104). PD$\mathrm{L} 1$ expression is driven by structural variations $(\mathrm{SV})$ on chromosomal PD-L1/L2 loci regions (105). Fluorescence in situ hybridization (FISH) can be used to identify PDL1 SVs (106). However, in lymphomas beyond cHL and PMBCL, there is no correlation between PD-L1 SVs and response. Tumor immunogenicity is regulated by several complex pathways and immune cells in the TME. Tumor mutational burden (TMB) is considered a biomarker of response in ICI patients. TMB is the total number of nonsynonymous mutations in the tumor (107). In one study, TMB was defined as low when there were less than or equal to 6 mutations per megabase $(\mathrm{mt} / \mathrm{Mb})$, intermediate TMB from 7 and $16 \mathrm{mt} / \mathrm{Mb}$, and high with greater than or equal to $17 \mathrm{mt} / \mathrm{Mb}$ (108). High levels of TMB in cHL and PCNSL were associated with favorable ORR and PFS (108). TMB expression in DLBCL, cHL and PCNSL 
tends to be very high while in SLL, PTCL, and NK/T cell lymphoma it is one of the lowest, thereby suggesting that the correlation with TMB may not be linear in lymphomas (109). Other potential biomarkers of ICIs are serum IFN- $\gamma$ levels and number of CD8-positive monocyte TILs on the tumor sample $(110,111)$. Soluble PD-L1 expression was positively correlated with increased IFN- $\gamma$ levels and poor prognosis in PTCL (112). PCNSL patients with elevated PD-L1 expression on tissue were also found to have a worse prognosis compared with those with low PD-L1 expression (113). The interplay between ICI use and upregulation of IFN- $\gamma$ needs further investigation to ascertain subgroups of patients that might benefit from the use of ICIs.

\section{Immune-related adverse events (irAEs) in lymphoma}

IrAEs reflect an "over-stimulated" immune system that can affect any body part and most commonly comprise dermatological (rash/dermatitis), gastrointestinal (colitis, hepatitis, pancreatitis), pulmonary (pneumonitis) and endocrine (thyroid, hypophysitis/adrenal crisis) sideeffects (114). With anti-CTLA-4 antibodies, irAEs are uncommon but one prevalent treatment related toxicity is diarrhea which occurs in $60 \%$ of cases and almost $30 \%$ are grade 3-4 (115). In allogeneic HSCT recipients, the use of ipilimumab did not cause any severe GvHD as evidenced in a small trial where $10 \%$ of patients developed a grade 3 chronic liver GvHD (116,117). In a large metaanalysis, about $15 \%$ of patients treated with $\mathrm{PD}-1 / \mathrm{PD}$ L1 ICIs were shown to develop irAEs and this increased to $60 \%$ in combinational ICI therapy with ipilimumab/ nivolumab (118). In a phase I study of r/r NHL (CA209039) treated with PD-1/PD-L1 antibodies, about $4 \%$ of patients developed grade 3-5 pneumonitis (21). One other less severe toxicity was fatigue which was mostly grade 1-2 and occurred in $10-50 \%$ of patients (13). In Keynote-089, patients with $\mathrm{r} / \mathrm{r}$ cHL post AHSCT received pembrolizumab with common irAEs including hypothyroidism (16\%), pneumonitis (5\%), and hyperthyroidism (4\%) (16). No grade 4 adverse events or treatment-related deaths were reported. Also, in a cohort of 14 patients that received nivolumab for $\mathrm{r} / \mathrm{r} \mathrm{cHL}$ after allogeneic SCT, acute GvHD was found in three patients with a prior history of GvHD (119). The management of irAEs from ICI therapy depends on the particular toxicity, and early recognition and institution of corticosteroids is critical to avoid permanent organ injury or patient mortality. Although corticosteroid use has been shown to reduce the efficacy of ICI therapy in various solid tumors, this finding has not been replicated in lymphoma studies yet.

\section{Future direction}

Although ICI therapy seems to have changed the treatment paradigm for the management of $\mathrm{cHL}$, we have not made many strides with ICI use in other lymphomas. Recently, there is some emerging evidence for potential efficacy of ICI therapy in PMBCL, PCNSL, and cutaneous T-cell lymphomas. Further, there is preliminary evidence of safety and efficacy with ICI use in HIV-associated lymphomas. However, larger studies with longer follow-up are awaited prior to making any practice changing recommendations. Further research is needed to identify the right patient and context of ICI use-either as monotherapy or as combinatorial therapy.

Indeed, ICI therapy has unique advantages and disadvantages compared to other forms of immunotherapy such as CAR-T cell therapy, which has already been approved for certain lymphomas. One obvious positive of ICI therapy is the low rate of severe toxicities, especially when used as monotherapy. Unless in the context of a clinical trial, ICI therapy is typically given to heavily pretreated patients who have undergone several cytotoxic chemotherapies. ICI therapy presents a well-tolerated approach for these patients. As seen in other malignancies, ICI therapy can also be very practically and safely combined with other anti- cancer agents, and a synergistic effect can be seen due to T-cell immunomodulation when ICI therapy is combined with other targeted or cytotoxic standard therapies (120). ICI therapies also tend to be associated with a durable response possibly, due to the mechanism of revitalizing a host's immune system. Obvious disadvantages to ICI therapy in lymphomas are that they seem to be only effective in certain types of lymphomas. Aggressive lymphomas such as DLBCL and BL often have barriers to ICI effectiveness, due to these tumor's defects in antigen presentation, a low immune cell microenvironment, TAM mediated immunosuppression, and genetic factors.

As aforementioned, with approvals of CAR-T in DLBCL and MCL, there may be value in evaluating the role of ICI use post CAR-T progression as those patients would have altered immune milieu favoring the use of ICI therapies. There are several areas of unmet need in lymphomas, and collaborations between basic scientists and clinical investigators are paramount to harnessing the immune 
system effectively and improve patient outcomes.

\section{Acknowledgments}

Funding: None.

\section{Footnote}

Provenance and Peer Review: This article was commissioned by the Guest Editors (Umang Swami and Mohammed M. Milhem) for the series "Cancer Immunotherapy: Recent Advances and Challenges" published in Annals of Translational Medicine. The article has undergone external peer review.

Peer Review File: Available at http://dx.doi.org/10.21037/ atm-20-6833

Conflicts of Interest: All authors have completed the ICMJE uniform disclosure form (available at http:// dx.doi.org/10.21037/atm-20-6833). The series "Cancer Immunotherapy: Recent Advances and Challenges" was commissioned by the editorial office without any funding or sponsorship. The authors have no other conflicts of interest to declare.

Ethical Statement: The authors are accountable for all aspects of the work in ensuring that questions related to the accuracy or integrity of any part of the work are appropriately investigated and resolved.

Open Access Statement: This is an Open Access article distributed in accordance with the Creative Commons Attribution-NonCommercial-NoDerivs 4.0 International License (CC BY-NC-ND 4.0), which permits the noncommercial replication and distribution of the article with the strict proviso that no changes or edits are made and the original work is properly cited (including links to both the formal publication through the relevant DOI and the license). See: https://creativecommons.org/licenses/by-nc-nd/4.0/.

\section{References}

1. Witkowska M, Smolewski P. Immune Checkpoint Inhibitors to Treat Malignant Lymphomas. J Immunol Res 2018;2018:1982423.

2. Salik B, Smyth MJ, Nakamura K. Targeting immune checkpoints in hematological malignancies. J Hematol
Oncol 2020;13:111.

3. Veldman J, Visser L, Berg AVD, et al. Primary and acquired resistance mechanisms to immune checkpoint inhibition in Hodgkin lymphoma. Cancer Treat Rev 2020;82:101931.

4. Kuzume A, Chi S, Yamauchi N, et al. Immune-Checkpoint Blockade Therapy in Lymphoma. Int J Mol Sci 2020;21:5456.

5. Eyre TA, Collins GP. Immune checkpoint inhibition in lymphoid disease. Br J Haematol 2015;170:291-304.

6. Zou W, Chen L. Inhibitory B7-family molecules in the tumour microenvironment. Nat Rev Immunol 2008;8:467-77.

7. Thanarajasingam G, Thanarajasingam U, Ansell SM. Immune checkpoint blockade in lymphoid malignancies. FEBS J 2016;283:2233-44.

8. Spagnuolo A, Gridelli C. "Comparison of the toxicity profile of PD-1 versus PD-L1 inhibitors in non-small cell lung cancer": is there a substantial difference or not? J Thorac Dis 2018;10:S4065-8.

9. Vaddepally RK, Kharel P, Pandey R, et al. Review of Indications of FDA-Approved Immune Checkpoint Inhibitors per NCCN Guidelines with the Level of Evidence. Cancers (Basel) 2020;12:738.

10. Ansell SM, Lesokhin AM, Borrello I, et al. PD-1 blockade with nivolumab in relapsed or refractory Hodgkin's lymphoma. N Engl J Med 2015;372:311-9.

11. Diefenbach CS, Connors JM, Friedberg JW, et al. Hodgkin Lymphoma: Current Status and Clinical Trial Recommendations. J Natl Cancer Inst 2016;109:djw249.

12. Herrera AF, Moskowitz AJ, Bartlett NL, et al. Interim results of brentuximab vedotin in combination with nivolumab in patients with relapsed or refractory Hodgkin lymphoma. Blood 2018;131:1183-94.

13. Ansell S, Gutierrez ME, Shipp MA, et al. A Phase 1 Study of Nivolumab in Combination with Ipilimumab for Relapsed or Refractory Hematologic Malignancies (CheckMate 039). Blood 2016;128:183.

14. Kasamon YL, de Claro RA, Wang Y, et al. FDA Approval Summary: Nivolumab for the Treatment of Relapsed or Progressive Classical Hodgkin Lymphoma. Oncologist 2017;22:585-91.

15. Armand P, Shipp MA, Ribrag V, et al. Programmed Death-1 Blockade With Pembrolizumab in Patients With Classical Hodgkin Lymphoma After Brentuximab Vedotin Failure. J Clin Oncol 2016;34:3733-9.

16. Chen R, Zinzani PL, Fanale MA, et al. Phase II Study of the Efficacy and Safety of Pembrolizumab for Relapsed/ 
Refractory Classic Hodgkin Lymphoma. J Clin Oncol 2017;35:2125-32.

17. Armand P, Nagler A, Weller EA, et al. Disabling immune tolerance by programmed death-1 blockade with pidilizumab after autologous hematopoietic stemcell transplantation for diffuse large B-cell lymphoma: results of an international phase II trial. J Clin Oncol 2013;31:4199-206.

18. Advani R, Flinn I, Popplewell L, et al. CD47 Blockade by Hu5F9-G4 and Rituximab in Non-Hodgkin's Lymphoma. N Engl J Med 2018;379:1711-21.

19. Ansell SM, Minnema MC, Johnson P, et al. Nivolumab for Relapsed/Refractory Diffuse Large B-Cell Lymphoma in Patients Ineligible for or Having Failed Autologous Transplantation: A Single-Arm, Phase II Study. J Clin Oncol 2019;37:481-9.

20. Herrera AF, Goy A, Mehta A, et al. Safety and activity of ibrutinib in combination with durvalumab in patients with relapsed or refractory follicular lymphoma or diffuse large B-cell lymphoma. Am J Hematol 2020;95:18-27.

21. Lesokhin AM, Ansell SM, Armand P, et al. Nivolumab in Patients With Relapsed or Refractory Hematologic Malignancy: Preliminary Results of a Phase Ib Study. J Clin Oncol 2016;34:2698-704.

22. Armand P, Rodig S, Melnichenko V, et al. Pembrolizumab in Relapsed or Refractory Primary Mediastinal Large B-Cell Lymphoma. J Clin Oncol 2019;37:3291-9.

23. Armand P, Kuruvilla J, Michot JM, et al. KEYNOTE-013 4-year follow-up of pembrolizumab in classical Hodgkin lymphoma after brentuximab vedotin failure. Blood Adv 2020;4:2617-22.

24. Zinzani PL, Santoro A, Gritti G, et al. Nivolumab Combined With Brentuximab Vedotin for Relapsed/ Refractory Primary Mediastinal Large B-Cell Lymphoma: Efficacy and Safety From the Phase II CheckMate 436 Study. J Clin Oncol 2019;37:3081-9.

25. Ding W, LaPlant BR, Call TG, et al. Pembrolizumab in patients with CLL and Richter transformation or with relapsed CLL. Blood 2017;129:3419-27.

26. Jain N, Basu S, Thompson PA, et al. Nivolumab Combined with Ibrutinib for CLL and Richter Transformation: A Phase II Trial. Blood 2016;128:59.

27. Westin JR, Chu F, Zhang M, et al. Safety and activity of PD1 blockade by pidilizumab in combination with rituximab in patients with relapsed follicular lymphoma: a single group, open-label, phase 2 trial. Lancet Oncol 2014;15:69-77.

28. Nastoupil LJ, Westin J, Fowler N, et al. High response rates with pembrolizumab in combination with rituximab in patients with relapsed follicular lymphoma: interim results of an on open-label, phase II study. Hematol Oncol 2017;35:120-1.

29. Palomba ML, Till BG, Park SI, et al. A phase Ib study evaluating the safety and clinical activity of atezolizumab combined with obinutuzumab in patients with relapsed or refractory non-Hodgkin lymphoma (NHL). Hematol Oncol 2017;35:137-8.

30. Khodadoust MS, Rook AH, Porcu P, et al. Pembrolizumab in Relapsed and Refractory Mycosis Fungoides and Sézary Syndrome: A Multicenter Phase II Study. Journal of Clinical Oncology 2020;38:20-8.

31. Kim SJ, Hyeon J, Cho I, et al. Comparison of Efficacy of Pembrolizumab between Epstein-Barr VirusPositive and Negative Relapsed or Refractory Non-Hodgkin Lymphomas. Cancer Res Treat 2019;51:611-22.

32. Kwong YL, Chan TSY, Tan D, et al. PD1 blockade with pembrolizumab is highly effective in relapsed or refractory NK/T-cell lymphoma failing l-asparaginase. Blood 2017;129:2437-42.

33. Uldrick TS, Goncalves PH, Abdul-Hay M, et al. Assessment of the Safety of Pembrolizumab in Patients With HIV and Advanced Cancer-A Phase 1 Study. JAMA Oncol 2019;5:1332-9.

34. Rogacheva Y, Popova M, Nekrasova A, et al. Case series of nivolumab treatment in patients with relapsed/refractory HIV-related lymphomas. Hematol Oncol 2019;37:500-2.

35. Merryman RW, Kim HT, Zinzani PL, et al. Safety and efficacy of allogeneic hematopoietic stem cell transplant after PD-1 blockade in relapsed/refractory lymphoma. Blood 2017;129:1380-8.

36. Holderried TAW, Fraccaroli A, Schumacher M, et al. The role of checkpoint blockade after allogeneic stem cell transplantation in diseases other than Hodgkin's Lymphoma. Bone Marrow Transplant 2019;54:1662-7.

37. Khouri IF, Fernandez Curbelo I, Turturro F, et al. Ipilimumab plus Lenalidomide after Allogeneic and Autologous Stem Cell Transplantation for Patients with Lymphoid Malignancies. Clin Cancer Res 2018;24:1011-8.

38. Kuruvilla J, Ramchandren R, Santoro A, et al. KEYNOTE-204: Randomized, open-label, phase III study of pembrolizumab (pembro) versus brentuximab vedotin (BV) in relapsed or refractory classic Hodgkin lymphoma (R/R cHL). J Clin Oncol 2020;38:8005.

39. Twa DD, Chan FC, Ben-Neriah S, et al. Genomic rearrangements involving programmed death ligands are recurrent in primary mediastinal large B-cell lymphoma. 
Blood 2014;123:2062-5.

40. Kiyasu J, Miyoshi H, Hirata A, et al. Expression of programmed cell death ligand 1 is associated with poor overall survival in patients with diffuse large B-cell lymphoma. Blood 2015;126:2193-201.

41. Song MK, Park BB, Uhm J. Understanding Immune Evasion and Therapeutic Targeting Associated with PD-1/ PD-L1 Pathway in Diffuse Large B-cell Lymphoma. Int J Mol Sci 2019;20:1326.

42. Tomassetti S, Chen R, Dandapani S. The role of pembrolizumab in relapsed/refractory primary mediastinal large B-cell lymphoma. Ther Adv Hematol 2019;10:2040620719841591.

43. Chapuy B, Roemer MG, Stewart C, et al. Targetable genetic features of primary testicular and primary central nervous system lymphomas. Blood 2016;127:869-81.

44. Muenst S, Hoeller S, Willi N, et al. Diagnostic and prognostic utility of PD-1 in B cell lymphomas. Dis Markers 2010;29:47-53.

45. Brusa D, Serra S, Coscia M, et al. The PD-1/PD-L1 axis contributes to T-cell dysfunction in chronic lymphocytic leukemia. Haematologica 2013;98:953-63.

46. Ramsay AG, Evans R, Kiaii S, et al. Chronic lymphocytic leukemia cells induce defective LFA-1-directed T-cell motility by altering Rho GTPase signaling that is reversible with lenalidomide. Blood 2013;121:2704-14.

47. Annibali O, Crescenzi A, Tomarchio V, et al. PD-1 /PDL1 checkpoint in hematological malignancies. Leuk Res 2018;67:45-55.

48. Nunes C, Wong R, Mason M, et al. Expansion of a CD8(+) $\mathrm{PD}-1(+)$ replicative senescence phenotype in early stage CLL patients is associated with inverted CD4:CD8 ratios and disease progression. Clin Cancer Res 2012;18:678-87.

49. Ramsay AG, Clear AJ, Fatah R, et al. Multiple inhibitory ligands induce impaired $\mathrm{T}$-cell immunologic synapse function in chronic lymphocytic leukemia that can be blocked with lenalidomide: establishing a reversible immune evasion mechanism in human cancer. Blood 2012;120:1412-21.

50. Qorraj M, Bruns H, Bottcher M, et al. The PD-1/PDL1 axis contributes to immune metabolic dysfunctions of monocytes in chronic lymphocytic leukemia. Leukemia 2017;31:470-8.

51. Armand P, Janssens AM, Gritti G, et al. Efficacy and safety results from CheckMate 140, a phase 2 study of nivolumab for relapsed/refractory follicular lymphoma. Blood 2021;137:637-45.

52. Harrington BK, Wheeler E, Hornbuckle K, et al. Modulation of immune checkpoint molecule expression in mantle cell lymphoma. Leuk Lymphoma 2019;60:2498-507.

53. Ok CY, Li L, Young KH. EBV-driven B-cell lymphoproliferative disorders: from biology, classification and differential diagnosis to clinical management. Exp Mol Med 2015;47:e132.

54. Vereide D, Sugden B. Insights into the evolution of lymphomas induced by Epstein-Barr virus. Adv Cancer Res 2010;108:1-19.

55. Barros MH, Segges P, Vera-Lozada G, et al. Macrophage polarization reflects $T$ cell composition of tumor microenvironment in pediatric classical Hodgkin lymphoma and has impact on survival. PLoS One 2015;10:e0124531.

56. Chuang HC, Lay JD, Hsieh WC, et al. Epstein-Barr virus LMP1 inhibits the expression of SAP gene and upregulates Th1 cytokines in the pathogenesis of hemophagocytic syndrome. Blood 2005;106:3090-6.

57. Green MR, Rodig S, Juszczynski P, et al. Constitutive AP-1 activity and EBV infection induce PD-L1 in Hodgkin lymphomas and posttransplant lymphoproliferative disorders: implications for targeted therapy. Clin Cancer Res 2012;18:1611-8.

58. Chen BJ, Chapuy B, Ouyang J, et al. PD-L1 expression is characteristic of a subset of aggressive B-cell lymphomas and virus-associated malignancies. Clin Cancer Res 2013;19:3462-73

59. Kataoka K, Miyoshi H, Sakata S, et al. Frequent structural variations involving programmed death ligands in Epstein-Barr virus-associated lymphomas. Leukemia 2019;33:1687-99.

60. Nicolae A, Pittaluga S, Abdullah S, et al. EBV-positive large B-cell lymphomas in young patients: a nodal lymphoma with evidence for a tolerogenic immune environment. Blood 2015;126:863-72.

61. Marra A, Scognamiglio G, Peluso I, et al. Immune Checkpoint Inhibitors in Melanoma and HIV Infection. Open AIDS J 2017;11:91-100.

62. Cubas RA, Mudd JC, Savoye AL, et al. Inadequate T follicular cell help impairs B cell immunity during HIV infection. Nat Med 2013;19:494-9.

63. Choi J, Goh G, Walradt T, et al. Genomic landscape of cutaneous T cell lymphoma. Nat Genet 2015;47:1011-9.

64. Bennani NN, Pederson LD, Atherton P, et al. A Phase II Study of Nivolumab in Patients with Relapsed or Refractory Peripheral T-Cell Lymphoma. Blood 2019;134:467. 
65. Saha A, Aoyama K, Taylor PA, et al. Host programmed death ligand 1 is dominant over programmed death ligand 2 expression in regulating graft-versus-host disease lethality. Blood 2013;122:3062-73.

66. Haverkos BM, Abbott D, Hamadani M, et al. PD-1 blockade for relapsed lymphoma post-allogeneic hematopoietic cell transplant: high response rate but frequent GVHD. Blood 2017;130:221-8.

67. Blazar BR, Carreno BM, Panoskaltsis-Mortari A, et al. Blockade of programmed death-1 engagement accelerates graft-versus-host disease lethality by an IFN-gammadependent mechanism. J Immunol 2003;171:1272-7.

68. Kline J, Godfrey J, Ansell SM. The immune landscape and response to immune checkpoint blockade therapy in lymphoma. Blood 2020;135:523-33.

69. Nijland M, Veenstra RN, Visser L, et al. HLA dependent immune escape mechanisms in B-cell lymphomas: Implications for immune checkpoint inhibitor therapy? Oncoimmunology 2017;6:e1295202.

70. Dubois S, Viailly PJ, Mareschal S, et al. Next-Generation Sequencing in Diffuse Large B-Cell Lymphoma Highlights Molecular Divergence and Therapeutic Opportunities: a LYSA Study. Clin Cancer Res 2016;22:2919-28.

71. Reichel J, Chadburn A, Rubinstein PG, et al. Flow sorting and exome sequencing reveal the oncogenome of primary Hodgkin and Reed-Sternberg cells. Blood 2015;125:1061-72.

72. Fangazio M, Dominguez-Sola D, Tabbò F, et al. Genetic Mechanisms of Immune Escape in Diffuse Large B Cell Lymphoma. Blood 2014;124:1692.

73. Steidl C, Shah SP, Woolcock BW, et al. MHC class II transactivator CIITA is a recurrent gene fusion partner in lymphoid cancers. Nature 2011;471:377-81.

74. God JM, Cameron C, Figueroa J, et al. Elevation of c-MYC disrupts HLA class II-mediated immune recognition of human B cell tumors. J Immunol 2015;194:1434-45.

75. Goebeler ME, Knop S, Viardot A, et al. Bispecific T-Cell Engager (BiTE) Antibody Construct Blinatumomab for the Treatment of Patients With Relapsed/Refractory NonHodgkin Lymphoma: Final Results From a Phase I Study. J Clin Oncol 2016;34:1104-11.

76. Zitvogel L, Galluzzi L, Smyth MJ, et al. Mechanism of action of conventional and targeted anticancer therapies: reinstating immunosurveillance. Immunity 2013;39:74-88.

77. de Charette M, Houot R. Hide or defend, the two strategies of lymphoma immune evasion: potential implications for immunotherapy. Haematologica 2018;103:1256-68.
78. Armand P. Immune checkpoint blockade in hematologic malignancies. Blood 2015;125:3393-400.

79. He Y, Rivard CJ, Rozeboom L, et al. Lymphocyteactivation gene-3, an important immune checkpoint in cancer. Cancer Sci 2016;107:1193-7.

80. Ruffo E, Wu RC, Bruno TC, et al. Lymphocyte-activation gene 3 (LAG3): The next immune checkpoint receptor. Semin Immunol 2019;42:101305.

81. Ghosh S, Sharma G, Travers J, et al. TSR-033, a Novel Therapeutic Antibody Targeting LAG-3, Enhances T-Cell Function and the Activity of PD-1 Blockade In Vitro and In Vivo. Mol Cancer Ther 2019;18:632-41.

82. Sakuishi K, Apetoh L, Sullivan JM, et al. Targeting Tim-3 and PD-1 pathways to reverse $T$ cell exhaustion and restore anti-tumor immunity. J Exp Med 2010;207:2187-94.

83. Garrido F, Aptsiauri N, Doorduijn EM, et al. The urgent need to recover $\mathrm{MHC}$ class $\mathrm{I}$ in cancers for effective immunotherapy. Curr Opin Immunol 2016;39:44-51.

84. Romano E, Kusio-Kobialka M, Foukas PG, et al. Ipilimumab-dependent cell-mediated cytotoxicity of regulatory $\mathrm{T}$ cells ex vivo by nonclassical monocytes in melanoma patients. Proc Natl Acad Sci U S A 2015;112:6140-5.

85. Zitvogel L, Apetoh L, Ghiringhelli F, et al. Immunological aspects of cancer chemotherapy. Nat Rev Immunol 2008;8:59-73.

86. Komohara Y, Niino D, Ohnishi K, et al. Role of tumorassociated macrophages in hematological malignancies. Pathol Int 2015;65:170-6.

87. Guo B, Cen H, Tan X, et al. Meta-analysis of the prognostic and clinical value of tumor-associated macrophages in adult classical Hodgkin lymphoma. BMC Med 2016;14:159.

88. Cebon J. Perspective: cancer vaccines in the era of immune checkpoint blockade. Mamm Genome 2018;29:703-13.

89. Allegra A, Russo S, Gerace D, et al. Vaccination strategies in lymphoproliferative disorders: Failures and successes. Leuk Res 2015;39:1006-19.

90. Brody J, Kohrt H, Marabelle A, et al. Active and passive immunotherapy for lymphoma: proving principles and improving results. J Clin Oncol 2011;29:1864-75.

91. Levy R, Ganjoo KN, Leonard JP, et al. Active idiotypic vaccination versus control immunotherapy for follicular lymphoma. J Clin Oncol 2014;32:1797-803.

92. Di Nicola M, Zappasodi R, Carlo-Stella C, et al. Vaccination with autologous tumor-loaded dendritic cells induces clinical and immunologic responses in indolent B-cell lymphoma patients with relapsed and measurable 
disease: a pilot study. Blood 2009;113:18-27.

93. Jeanbart L, Ballester M, de Titta A, et al. Enhancing efficacy of anticancer vaccines by targeted delivery to tumor-draining lymph nodes. Cancer Immunol Res 2014;2:436-47.

94. Zhang H, Conrad DM, Butler JJ, et al. Adenosine acts through A2 receptors to inhibit IL-2-induced tyrosine phosphorylation of STAT5 in T lymphocytes: role of cyclic adenosine 3',5'-monophosphate and phosphatases. J Immunol 2004;173:932-44.

95. Holmgaard RB, Zamarin D, Munn DH, et al. Indoleamine 2,3-dioxygenase is a critical resistance mechanism in antitumor T cell immunotherapy targeting CTLA-4. J Exp Med 2013;210:1389-402.

96. Prendergast GC, Malachowski WP, DuHadaway JB, et al. Discovery of IDO1 Inhibitors: From Bench to Bedside. Cancer Res 2017;77:6795-811.

97. Wang X, Huang H, Young KH. The PTEN tumor suppressor gene and its role in lymphoma pathogenesis. Aging (Albany NY) 2015;7:1032-49.

98. Burr ML, Sparbier CE, Chan KL, et al. An Evolutionarily Conserved Function of Polycomb Silences the MHC Class I Antigen Presentation Pathway and Enables Immune Evasion in Cancer. Cancer Cell 2019;36:385-401.e8.

99. Ennishi D, Jiang A, Boyle M, et al. Double-Hit Gene Expression Signature Defines a Distinct Subgroup of Germinal Center B-Cell-Like Diffuse Large B-Cell Lymphoma. J Clin Oncol 2019;37:190-201.

100. Cesano A, Warren S. Bringing the next Generation of Immuno-Oncology Biomarkers to the Clinic. Biomedicines 2018;6:14.

101. Wright J. Epigenetics: reversible tags. Nature 2013;498:S10-1.

102.Xu-Monette ZY, Zhou J, Young KH. PD-1 expression and clinical PD-1 blockade in B-cell lymphomas. Blood 2018;131:68-83.

103.Perrier A, Didelot A, Laurent-Puig P, et al. Epigenetic Mechanisms of Resistance to Immune Checkpoint Inhibitors. Biomolecules 2020;10:1061.

104.Joos S, Otano-Joos MI, Ziegler S, et al. Primary mediastinal (thymic) B-cell lymphoma is characterized by gains of chromosomal material including $9 \mathrm{p}$ and amplification of the REL gene. Blood 1996;87:1571-8.

105. Roemer MG, Advani RH, Ligon AH, et al. PD-L1 and PD-L2 Genetic Alterations Define Classical Hodgkin Lymphoma and Predict Outcome. J Clin Oncol 2016;34:2690-7.

106. Green MR, Monti S, Rodig SJ, et al. Integrative analysis reveals selective 9p24.1 amplification, increased PD-1 ligand expression, and further induction via JAK2 in nodular sclerosing Hodgkin lymphoma and primary mediastinal large B-cell lymphoma. Blood 2010;116:3268-77.

107.Alexandrov LB, Nik-Zainal S, Wedge DC, et al. Signatures of mutational processes in human cancer. Nature 2013;500:415-21.

108. Ou A, Sumrall A, Phuphanich S, et al. Primary CNS lymphoma commonly expresses immune response biomarkers. Neurooncol Adv 2020;2:vdaa018.

109.Zhou H, Du X, Zhao T, et al. Distribution and influencing factors of tumor mutational burden in different lymphoma subtypes. J Clin Oncol 2019;37:e19053.

110. Ahmadzadeh M, Johnson LA, Heemskerk B, et al. Tumor antigen-specific CD8 $\mathrm{T}$ cells infiltrating the tumor express high levels of PD-1 and are functionally impaired. Blood 2009;114:1537-44.

111. Castro F, Cardoso AP, Goncalves RM, et al. InterferonGamma at the Crossroads of Tumor Immune Surveillance or Evasion. Front Immunol 2018;9:847.

112.Zhang X, Liu L, Zhou S, et al. Plasma soluble programmed death ligand 1 levels predict clinical response in peripheral T-cell lymphomas. Hematol Oncol 2019;37:270-6.

113. Kim S, Nam SJ, Park C, et al. High tumoral PD-L1 expression and low PD-1(+) or CD8(+) tumor-infiltrating lymphocytes are predictive of a poor prognosis in primary diffuse large B-cell lymphoma of the central nervous system. Oncoimmunology 2019;8:e1626653.

114. Bajwa R, Cheema A, Khan T, et al. Adverse Effects of Immune Checkpoint Inhibitors (Programmed Death-1 Inhibitors and Cytotoxic T-Lymphocyte-Associated Protein-4 Inhibitors): Results of a Retrospective Study. J Clin Med Res 2019;11:225-36.

115.Ansell SM, Hurvitz SA, Koenig PA, et al. Phase I study of ipilimumab, an anti-CTLA-4 monoclonal antibody, in patients with relapsed and refractory B-cell non-Hodgkin lymphoma. Clin Cancer Res 2009;15:6446-53.

116. Bashey A, Medina B, Corringham S, et al. CTLA4 blockade with ipilimumab to treat relapse of malignancy after allogeneic hematopoietic cell transplantation. Blood 2009;113:1581-8.

117. Davids MS, Kim HT, Costello CL, et al. A Multicenter Phase I/Ib Study of Ipilimumab for Relapsed Hematologic Malignancies after Allogeneic Hematopoietic Stem Cell Transplantation. Blood 2015;126:860.

118. Wang DY, Salem JE, Cohen JV, et al. Fatal Toxic Effects Associated With Immune Checkpoint Inhibitors: A 
Systematic Review and Meta-analysis. JAMA Oncol 2018;4:1721-8.

119. Herbaux C, Gauthier J, Brice P, et al. Nivolumab Is Effective and Reasonably Safe in Relapsed or Refractory Hodgkin's Lymphoma after Allogeneic Hematopoietic

Cite this article as: Hatic H, Sampat D, Goyal G. Immune checkpoint inhibitors in lymphoma: challenges and opportunities. Ann Transl Med 2021;9(12):1037. doi: 10.21037/ atm-20-6833
Cell Transplantation: A Study from the Lysa and SFGMTC. Blood 2015;126:3979.

120. Yan Y, Kumar AB, Finnes H, et al. Combining Immune Checkpoint Inhibitors With Conventional Cancer Therapy. Front Immunol 2018;9:1739. 